\title{
CONDYLO-CEPHALIC NAIL FIXATION FOR TROCHANTERIC FRACTURES OF THE FEMUR*
}

\author{
F. Collado, J. Vila and J. E. Beltrán, Barcelona, Spain \\ From the "Centro de Rehabilitación y Traumatologia", Barcelona
}

The intramedullary condylo-cephalic nail for the treatment of trochanteric fractures of the femur was introduced by Küntscher in 1966. The purpose of this paper is to present the results in our first hundred patients treated in the course of the two years from September 1969 to August 1971. By the time of writing in October 1972 the total had risen to 210 .

\section{TECHNIQUE}

The condylo-cephalic nail has a clover-leaf cross-section, a slight curve and a standard diameter of 10 millimetres. The lengths vary; we have used a range of 34 to 44 centimetres.

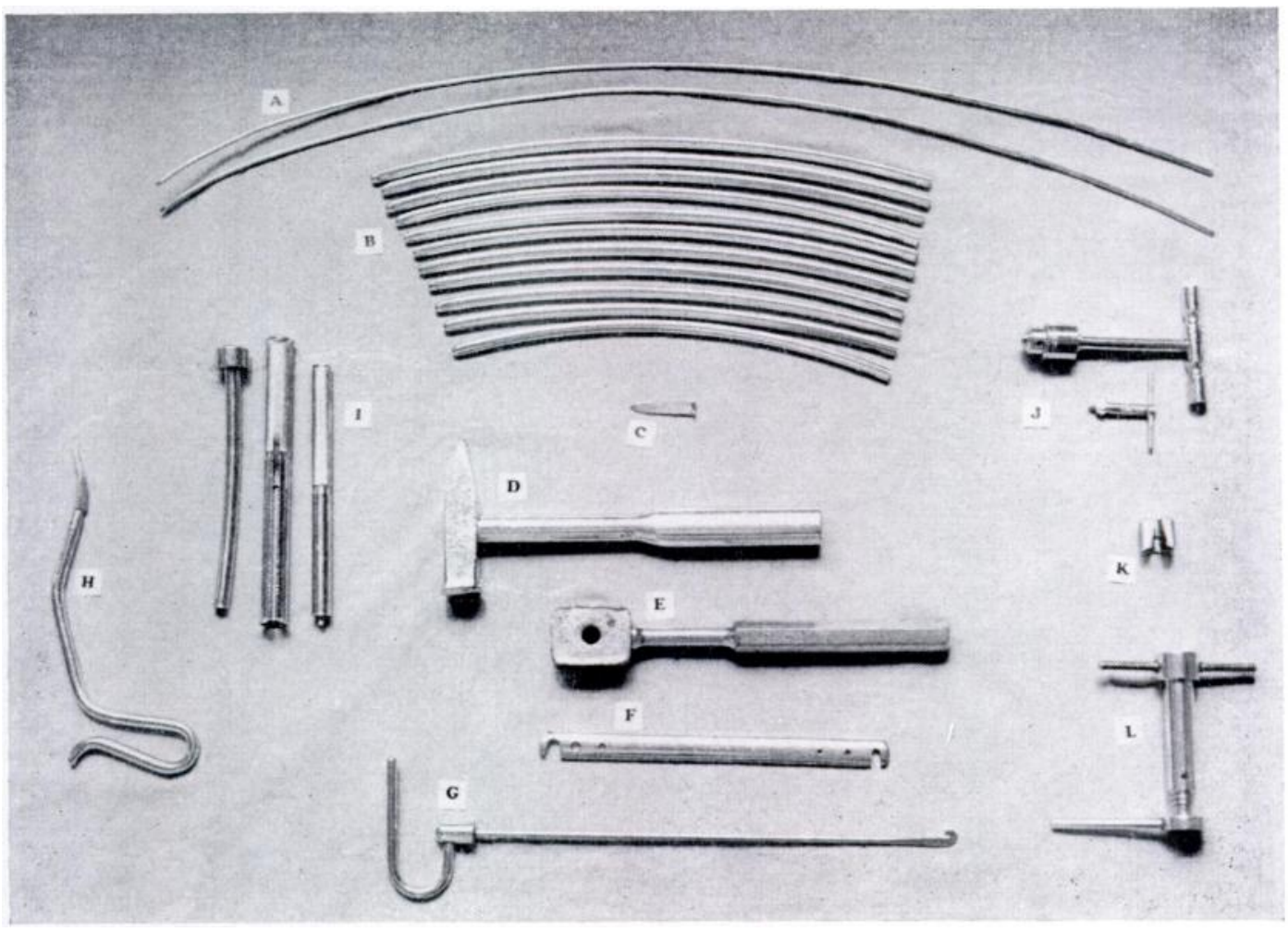

Fig. 1

To show the standard instrumentation. A-guide-wires; B-nails; C-"stopper"; D-hammer; E-mallet; F-wire bender; G-nail extractor; H-curved awl; I-punches; J-introducer with Jacob's chuck and tightener; $\mathbf{K}$-guard for chuck; L-guide wire extractor.

It is best to prepare a full range of nails, because it is difficult to assess the required length before operation. The armamentarium for the actual introduction is basic and simple, consisting only of a curved bone awl, a guide wire, an introducer with a Jacob's chuck and a mallet (Fig. 1).

* Based on a paper read to the British Orthopaedic Association at Aviemore in April 1972. 
The patient under general anaesthesia is placed on the orthopaedic table. Reduction is carried out in the usual manner and checked with the image intensifier. A skin incision two or three centimetres long is made over the medial condyle and deepened down to the area of insertion of the capsule. The bone is perforated with the curved awl at the junction of metaphysis and condyle (Fig. 2).
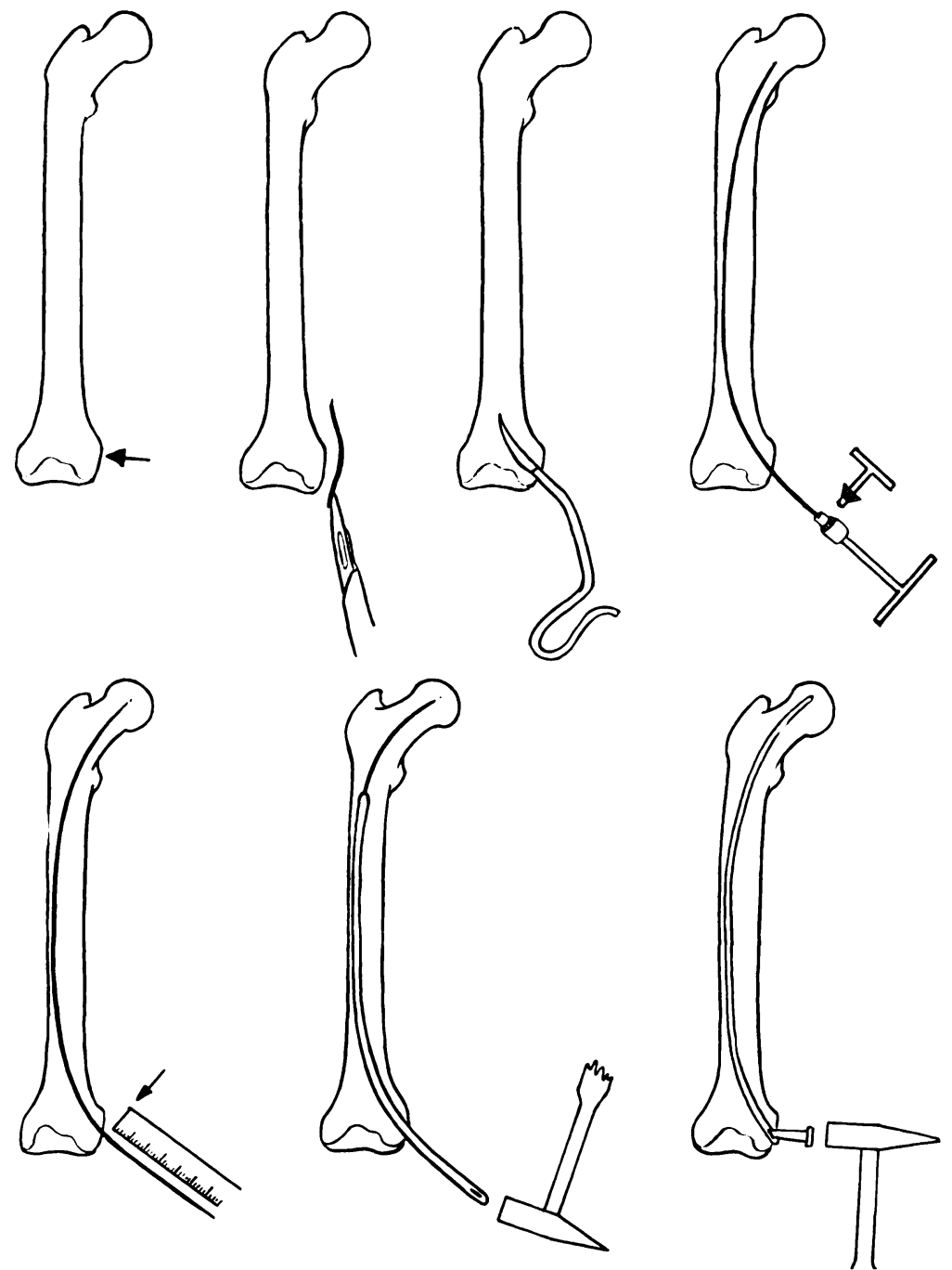

Fig. 2

Diagrams to illustrate the technique of insertion of guide-wire, nail and stopper.

(After Collado and Mijaers 1969.)

The guide wire, 85 centimetres in length, is introduced with the help of the Jacob's chuck, which is tightened 50 to 60 centimetres from its point. Rotation of the wire is controlled without difficulty, and it is pushed upwards towards the anterior aspect of the femoral neck. The passage of the guide wire in the medulla is followed with the aid of the image intensifier in both antero-posterior and lateral projections. When the lateral view shows posterior displacement of the distal fragment, the assistant aids reduction by pushing the upper third of the thigh forward. Once the point of the guide wire is in the hard nucleus of the head and one centimetre from the articular cartilage, the length of nail required is obtained by subtraction of the length of wire still exposed.

VOL. 55 B, NO. 4, NOVEMBER 1973 
The selected nail is introduced by light tapping of the mallet. Once it has passed through the fracture site, the traction is released in order to favour impaction. The end of the nail should come to rest one centimetre from the joint (Fig. 3). The guide wire is now extracted with the aid of the Jacob's chuck and special extractor. The slit in the distal end of the nail is exposed and a "stopper" consisting of a short flat pin is tapped into it (Fig. 4) in order to avoid any shift towards the knee. A few stitches suffice to close the incision.

Twenty-four hours after operation the patient is allowed to sit out of bed, and by four to eight days is encouraged to walk with two sticks.

\section{MATERIAL}

We have studied our first hundred consecutive cases of trochanteric fracture treated by this method. There were twenty-three men and seventy-seven women. In fifteen patients the ages were from sixty to seventy years, in thirty-eight from seventy-one to eighty, in thirty-nine from eighty-one to ninety, and in seven from ninety-one to 100 . For convenience we are adding to the first group a man aged thirty-five in poor general condition due to alcoholic coma.

The fracture was more common on the right side ( 59 per cent) than on the left ( 41 per cent). The line of fracture was pertrochanteric in seventy-six cases, basicervical in seventeen and subtrochanteric in seven (Fig. 5). Three fractures were sustained in road traffic accidents, the rest being due to falls. Seventy-five fractures occurred in the home and twenty-two outside. In no case was the fracture pathological because of metastasis or other disorder of bone, apart from senile decalcification. (In the case of a pathological fracture, internal fixation is performed using Müller's nail-plate, with or without cement, and a specimen for biopsy is taken, which is not possible with routine condylo-cephalic nailing.)

The general condition of the patient was considered good in 23 per cent, fair in 31 per cent and poor in 46 per cent. As general rule we considered a poor general condition as an indication for early operation. Indeed, recovery was more satisfactory in those patients on whom the operation was carried out soon after the accident.

On the patient's arrival in the Casualty Department we did not attempt formal reduction of the fracture as a routine measure. In eighty-two cases trans-tibial skeletal traction by Kirschner wire was applied under local anaesthesia, in twelve only a below-knee anti-rotation plaster was used, and in three a Braun splint without traction; the remaining three patients had simple bed rest before operation.

Out of the 100 patients nineteen died, all except one being over eighty years of age. Ten of the nineteen died during the first two weeks after operation, four from pulmonary embolism and six from cardiac failure or infection of the respiratory or urinary tract. Another three died between two and four weeks, four between four and eight weeks, and two between eight and sixteen weeks after the operation. The causes of death in this group were urinary or respiratory infection. Six patients had bed-sores.

Taking into account these nineteen deaths, the time in hospital was ten to twenty days for sixty-two patients, twenty to thirty days for eighteen, and over thirty days for twenty. In the first half of this series, we were more cautious in allowing the patients to bear weight, but once we saw that there was little risk of secondary displacement of either fracture or nail, we changed our policy and allowed partial weight-bearing four to eight days after the operation and full weight-bearing without sticks at six weeks.

We have had few complications with this procedure. In no case did the guide wire bend or break. There were no wound infections. Five patients had a small serous collection at the site of the incision, all treated by aspiration. In six cases the first radiographs after operation showed a slight degree of diastasis of the fracture which corrected itself as soon as the patient began to bear weight. In no case did the nail bend or break. None of the 100 patients required reinsertion of the nail because of poor reduction. There was no case of important secondary 
angular displacement when weight-bearing was begun, such as happens with other methods of internal fixation.

Nine nails projected a few millimetres through the head of the femur. Two projected very little and caused no discomfort; the other seven nails and their flat "stoppers" were removed without difficulty between four and seven months after insertion (Fig. 6). One

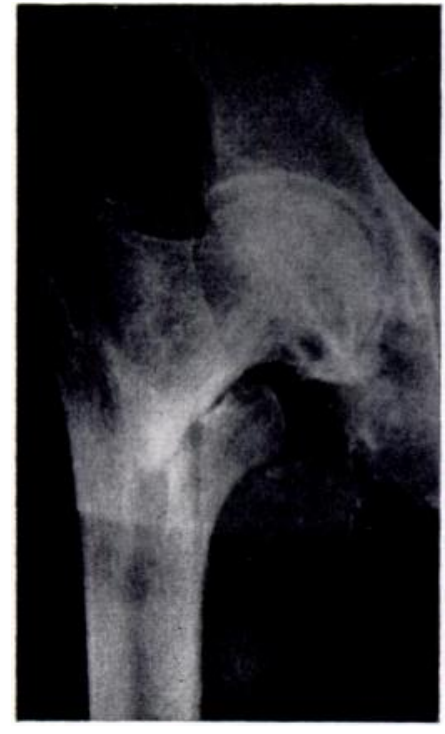

To show a pertrochanteric fracture in varus, with sound union in slight valgus after internal fixation by condylo-cephalic nail.
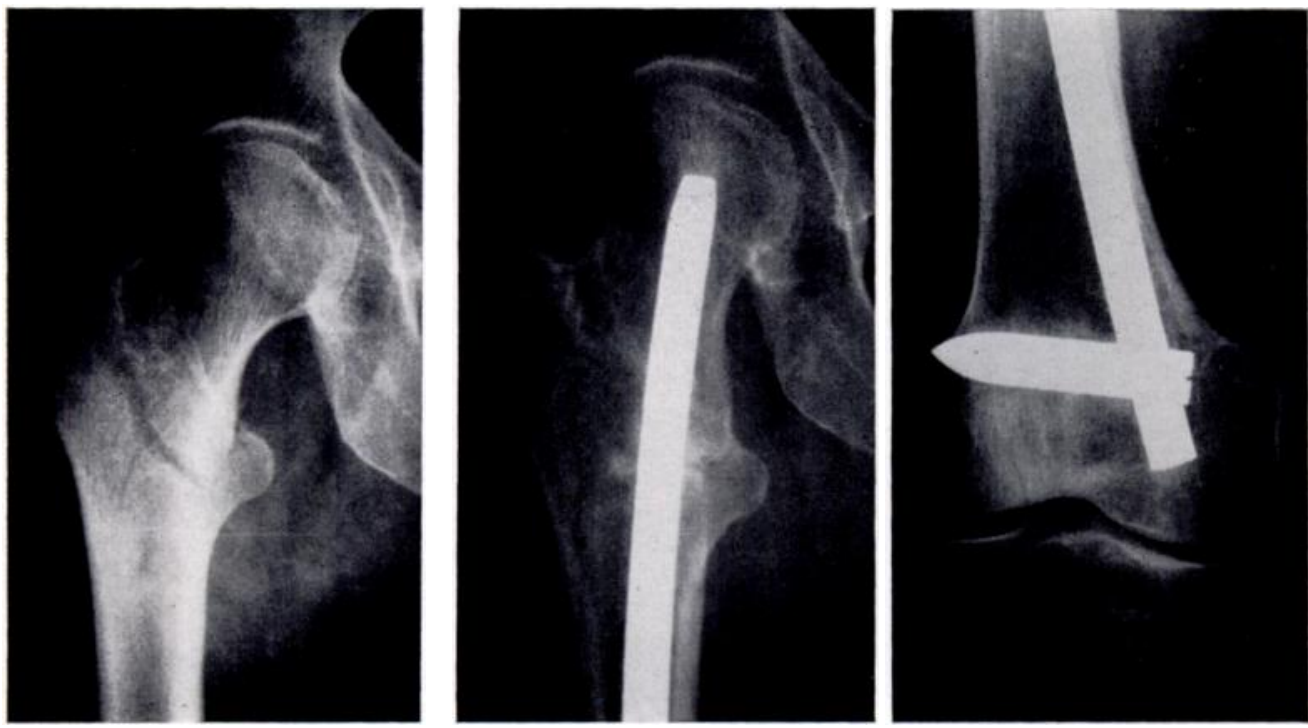

Fig. 4

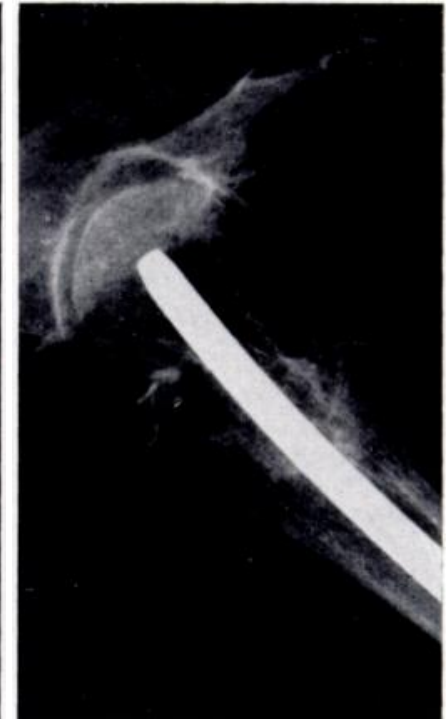

valgus after intermal fixation by

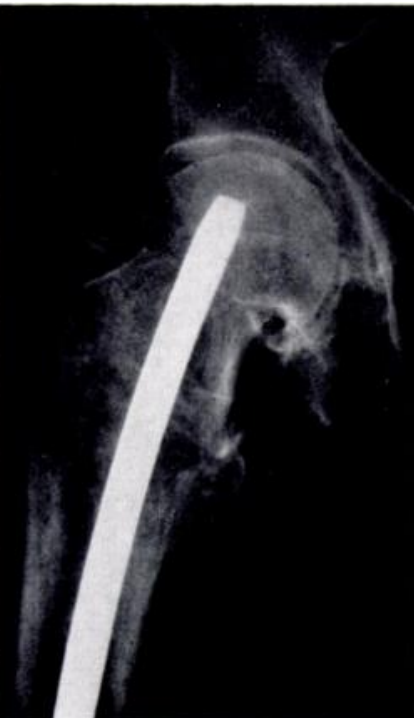

To show a pertrochanteric fracture with slight displacement, union in valgus, and the lower end of the femur with nail and stopper.

patient sustained a linear crack in the distal metaphysis of the femur which soon healed without special treatment. (After having no such trouble in the present series, in the last 110 cases there have been two instances of the nail receding into the knee joint and two of its cutting through the trochanter).

VOL. 55 B, NO. 4, NOVEMBER 1973 
In thirty cases the nail fixation was performed in valgus. This did not hamper the final results; the fractures united satisfactorily and the patients walked without difficulty; resorption of bone at the fracture site appeared to compensate for any elongation of the limb. In the other seventy cases the angle of the neck was practically normal or very slightly increased. No fracture was fixed in varus because the shape of the nail does not allow this.

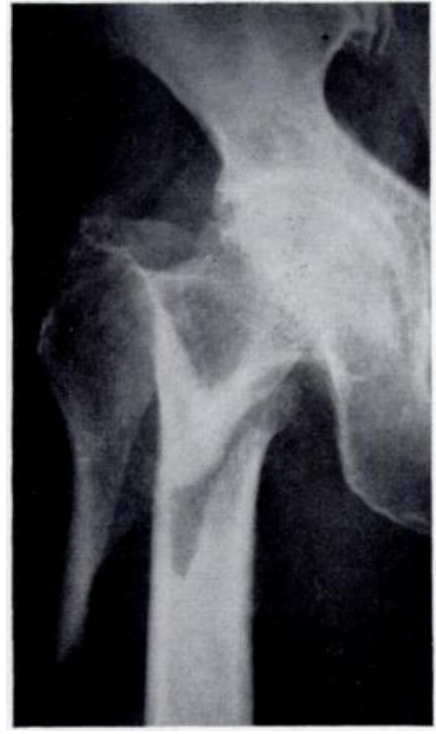

FIG. 5

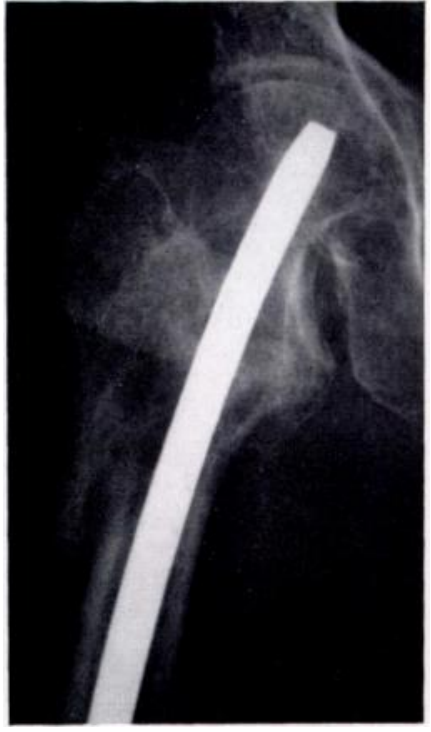

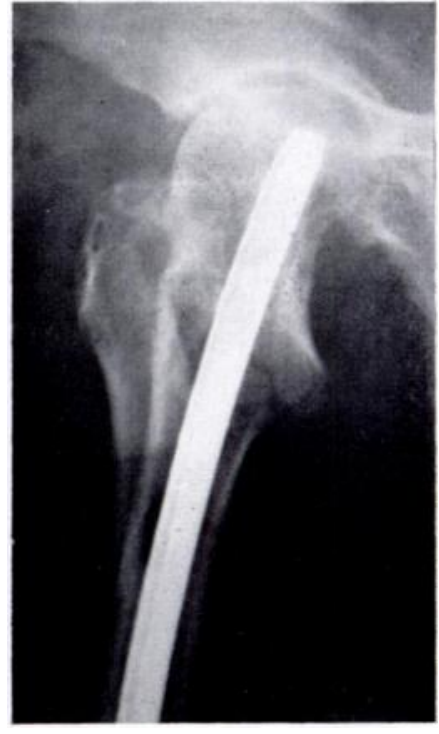

union secured in valgus.
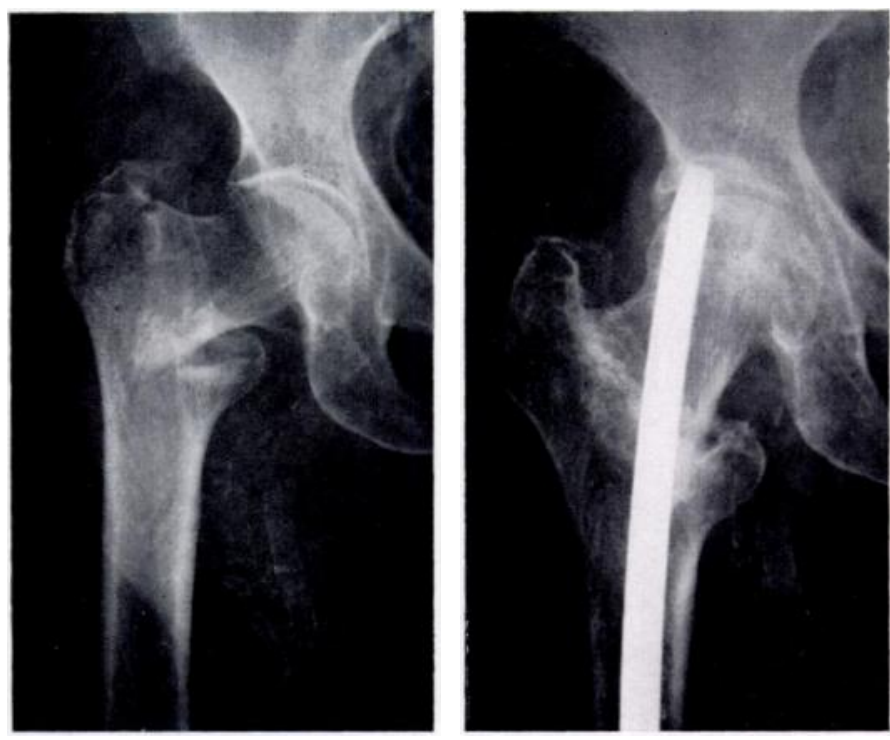

FIG. 6

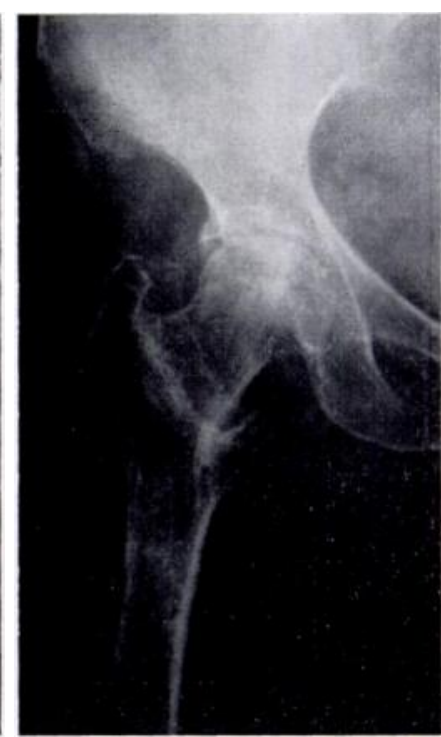

To show a pertrochanteric fracture in marked varus, with union secured by a nail which was 1 centimetre too long and was removed four months after operation.

Follow-up-Of the eighty-one patients alive, we have been unable to complete the files of sixteen because they were transferred to other hospitals or moved to other towns. For the remaining sixty-five patients, nineteen began partial weight-bearing with two sticks between one and two weeks after operation, twenty-eight between two and four weeks, eight between 
five and eight weeks, and ten by three months. Full weight-bearing was resumed by four patients in the first month, by thirteen between one and two months, by twenty-six between two and three months, and by fifteen between three and four months after operation; the remaining seven patients were still using two crutches at the time of the last examination.

Evaluation-We have graded the results in sixty-five patients taking into account such factors as pain, limp, the use of sticks or crutches and the patient's impression of the final result. The grading comes out as follows: very good (practically no after-effects, patient well satisfied), twenty-one; good (no crutches or one crutch out of doors, some trouble with hip or knee), thirty; fair (still needing two crutches), twelve; and bad (unable to walk unless assisted), two.

\section{DISCUSSION}

The main advantage of the condylo-cephalic nail is the solidity of the complex it forms with the two bone fragments, which in our opinion is superior to that obtained by other methods of treatment. This solidity is derived from two sources: 1) the nail is close to the long axis of the shaft, a position which is most favourable because it corresponds with the direction of the mechanical forces acting on the fracture line; and 2) the tendency to valgus permits uniform compression of the fracture surfaces when weight-bearing is commenced.

There are several other advantages. The material and instruments necessary for this method of osteosynthesis are simple, easily maintained and replaced, and inexpensive, apart from the television image intensifier. The surgical procedure is simple, taking no more than fifteen minutes; the short time under general anaesthesia, the minimal blood loss and the low risk of infection are also matters of considerable importance in these elderly patients. Because the incision is remote from the fracture site, the intensifier does not impede the surgeon and the radiation danger is minimal. The patient can bear weight early and under favourable circumstances can return home after a few days in hospital. Finally, because the nail is intramedullary, surface intolerance of the kind so common with pin and plate fixation is practically unknown.

\section{SUMMARY}

1. An initial series of 100 consecutive cases of trochanteric fracture treated by the Küntscher technique of condylo-cephalic nailing with the aid of an image intensifier has been reviewed.

2. The advantages of this simple method over internal fixation by open reduction and insertion of some kind of nail and plate are discussed.

\section{REFERENCES}

Collado, H., and Mujaers, G. (1969): El enclavijamiento de Küntscher en las fracturas pertrocantéreas. Sección Cirugía. Anales de Medicina, 55, 578.

KüNTSCHER, G. (1970): A New Method of Treatment of Pertrochanteric Fractures. Proceedings of the Royal Society of Medicine, 63, 1120. 